\section{MS9-O2 At play in the briar patch of epigenetics}

Jim Kiefer ${ }^{1}$, Patrick Trojer ${ }^{2}$, Marie Classon ${ }^{1}$, Maia Vinogradova ${ }^{1}$, Victor Gehling ${ }^{2}$, Shilpi Arora ${ }^{2}$, Amy Gustafson ${ }^{1}$, Brian Albrecht ${ }^{2}$, Charles Tindell ${ }^{1}$, Kaylyn Williamson ${ }^{2}$, Catherine Wilson ${ }^{1}$, Jennifer Busby ${ }^{2}$, Yichin Liu ${ }^{1}$, Pranoti Gangurde ${ }^{2}$, David Arnott ${ }^{1}$, Shane Buker ${ }^{2}$, Tommy Cheung ${ }^{1}$, Fei Lan ${ }^{2}$, Erica Jackson ${ }^{1}$, Megan Flynn $^{1}$, Andrea Cochran ${ }^{1}$, Tobias Maille ${ }^{1}$, Gulfem Guler ${ }^{1}$, Christopher Bailey ${ }^{2}$, Richard Cummings ${ }^{2}$, Robert Pitti ${ }^{1}$, Matthew Wongchenko ${ }^{1}$, Yibing Yang ${ }^{1}$, Ted Lau ${ }^{1}$, Mike Costa ${ }^{1}$, Jean-Christophe Harmange ${ }^{2}$, Jeffrey Settleman ${ }^{1}$

1. Genentech Inc., South San Francisco, California, USA

2. Constellation Pharmaceuticals, Cambridge, Massachusetts, USA

email: kiefer.james@gene.com

The understanding of non-genetic mechanisms in cancer biology and treatment resistance has steadily evolved over the last two decades. Several epigenetic modulator and monitoring proteins have been implicated in maintaining tumor cells in pluripotent - and often drug resistant - states. We have discovered a series of inhibitors that modulate epigenetic signaling and impact drug resistance. We used a multi-pronged approach to identify and optimize these molecules, including the determination of novel crystal structures of the target protein. In addition to aiding chemical design, these structures inform models for substrate recognition not previously possible in other systems.

Keywords: epigenetics, crystallography, inhibitor, cancer, structure based drug design

\section{MS9-03 Identification of novel allosteric inhibitors through Fragment-Based Drug Discovery and X-ray crystallography}

Puja Pathuri ${ }^{1}$, Susanne M. Saalau-Bethell ${ }^{1}$, Andrew J. Woodhead $^{1}$, Valerio Berdini ${ }^{1}$, Maria G. Carr ${ }^{1}$, Gianni Chessari ${ }^{1}$, Anne Cleasby ${ }^{1}$, Miles Congreve ${ }^{1}$, Joseph E. Coyle ${ }^{1}$, Brent

Graham $^{1}$, Steven D. Hiscock ${ }^{1}$, Victoria Lock ${ }^{1}$, Christopher W.

Murray $^{1}$, M. Alistair O’Brien ${ }^{1}$, Sharna J. Rich ${ }^{1}$, Caroline J.

Richardson ${ }^{1}$, Tracey Sambrook ${ }^{1}$, Mladen Vinkovic ${ }^{1}$, Pamela A. Williams ${ }^{1}$, Jeff R. Yon ${ }^{1}$, Harren Jhoti ${ }^{1}$

1. Astex Pharmaceuticals, 436 Cambridge Science Park, Milton Road, Cambridge, United Kingdom

email: Puja.Pathuri@astx.com

X-ray crystallography provides a powerful and sensitive primary screening technique for fragment-based drug discovery with the potential to detect binding events not only at precedented active sites, but also in previously unexploited pockets. Fragment-based drug discovery at Astex uses a combination of X-ray crystallography and other biophysical techniques including NMR, ITC and thermal shift $\left(\mathrm{T}_{\mathrm{m}}\right)$ to identify initial fragment hits at known binding sites and novel allosteric sites. Using our proprietary fragment screening platform, Pyramid ${ }^{\mathrm{TM}}$ we have successfully discovered molecules that bind at novel allosteric sites in different enzyme families. During this presentation we will show how we used fragment based drug discovery in the identification of significant allosteric sites on the full length NS3 protein from the Hepatitis C Virus (HCV) and human soluble Adenylate Cyclase.

Keywords: Fragment-based drug discovery, crystallography, allosteric sites 